\title{
artigo
}

Alecrin, E.S.; Guimarães, N.S.; Oliveira, A.L.G.; Lyon, S.; Martins, M.A.P.; Rocha, M.O.C.

Fatores de risco associados ao adoecimento em contatos de hanseníase: um protocolo de revisão sistemática

\section{Fatores de risco associados ao adoecimento em contatos de hanseníase: um protocolo de revisão sistemática}

\author{
Risk factors associated with illness in leprosy contacts: a systematic review protocol \\ Factores de riesgo asociados con la enfermedad en contactos con lepra: un protocolo de revisión sistemática
}

\begin{abstract}
RESUMO
Objetivo: Esta revisão terá como objetivo avaliar os fatores de risco associados ao adoecimento em contatos de hanseníase. Método: Estudos de caso-controle, transversais e de coorte, estudos prospectivos e retrospectivos, estudos experimentais, de janeiro de 2004 a dezembro de 2020. Os participantes serão contatos de hanseníase. As bases de dados eletrônicas incluirão BVS, PubMed, Cochrane Library, Embase. Estratégias de busca serão utilizadas empregando descritores para cada base de dados. Os artigos serão selecionados após leitura do título e resumo por dois revisores independentes. Após a leitura na íntegra, os artigos serão selecionados de acordo com os critérios de inclusão. Resultado: Os dados serão extraídos e tabulados para síntese narrativa. Conclusão: Monitorar os contatos é uma das estratégias mais eficazes para o controle da hanseníase. A identificação dos fatores de risco para adoecimento nos contatos pode contribuir para o desenvolvimento de ações em saúde pública. Número de registro da revisão sistemática: PROSPERO CRD4 2020160680

DESCRITORES: Hanseníase; Contatos com hanseníase; Doenças negligenciadas; Vigilância em saúde pública; Fatores de risco.
\end{abstract}

\section{ABSTRACT}

Objective: This review will aim to assess the risk factors associated with illness in leprosy contacts. Method: Case-control, cross-sectional and cohort studies, prospective and retrospective studies, experimental studies, from January 2004 to December 2020. Participants will be leprosy contacts. The electronic databases will include VHL, PubMed, Cochrane Library, Embase. Search strategies will be used employing descriptors for each database. The articles will be selected after reading the title and abstract by two independent reviewers. After reading in full, the articles will be selected according to the inclusion criteria. Result: Data will be extracted and tabulated for narrative synthesis. Conclusion: Monitoring contacts is one of the most effective strategies for leprosy control. The identification of risk factors for illness in contacts can contribute to the development of actions in public health. Systematic review registration number: PROSPERO CRD4 2020160680

DESCRIPTORS: Leprosy; Contacts with leprosy; Neglected diseases; Public health surveillance; Risk factors.

\section{RESUMEN}

Objetivo: Esta revisión tendrá como objetivo evaluar los factores de riesgo asociados con la enfermedad en los contactos con lepra. Método: Estudios de casos y controles, transversales y de cohortes, estudios prospectivos y retrospectivos, estudios experimentales, de enero de 2004 a diciembre de 2020. Los participantes serán contactos leprosos. Las bases de datos electrónicas incluirán BVS, PubMed, Cochrane Library, Embase. Se utilizarán estrategias de búsqueda empleando descriptores para cada base de datos. Los artículos serán seleccionados después de leer el título y el resumen por dos revisores independientes. Después de la lectura completa, los artículos se seleccionarán de acuerdo con los criterios de inclusión. Resultado: los datos se extraerán y tabularán para la síntesis narrativa. Conclusión: El seguimiento de los contactos es una de las estrategias más eficaces para el control de la lepra. La identificación de factores de riesgo de enfermedad en los contactos puede contribuir al desarrollo de acciones en salud pública. Número de registro de revisión sistemática: PROSPERO CRD4 2020160680

DESCRIPTORES: Lepra; Contactos con lepra; Enfermedades desatendidas; Vigilancia de la salud pública; Factores de riesgo.

RECEBIDO EM: 30/04/2021 APROVADO EM: 24/05/2021

\section{Edilamar Silva de Alecrin}

Universidade Federal de Minas Gerais, Pós-Graduação em Ciências da Saúde: Infectologia e Medicina Tropical, Belo Horizonte, Minas Gerais, Brazil. Hospital Eduardo de Menezes, Ambulatório de Dermatologia, Belo Horizonte, Minas Gerais, Brazil. Faculdade de Saúde e Ecologia Humana (FASEH), Vespasiano, Minas Gerais, Brazil.

ORCID: 0000-0003-0787-5259 


\section{Nathália Sernizon Guimarães}

Programa de Pós-Graduação em Saúde e Nutrição. Escola de Nutrição. Universidade Federal de Ouro Preto, Ouro Preto, Minas Gerais, Brazil.

ORCID: 0000-0002-0487-0500

\section{Ana Laura Grossi de Oliveira}

Universidade Federal de Minas Gerais, Pós-Graduação em Ciências da Saúde: Infectologia e Medicina Tropical, Belo Horizonte, Minas Gerais, Brazil.

ORCID: 0000-0003-4614-7502

\section{Sandra Lyon}

Hospital Eduardo de Menezes, Ambulatório de Dermatologia, Belo Horizonte, Minas Gerais, Brazil. Faculdade de Saúde e Ecologia Humana (FASEH), Vespasiano, Minas Gerais, Brazil.

ORCID: 0000-0001-8777-5068

\section{Maria Auxiliadora Parreiras Martins}

Universidade Federal de Minas Gerais, Pós-Graduação em Ciências da Saúde: Infectologia e Medicina Tropical, Belo Horizonte, Minas Gerais, Brazil.

ORCID: 0000-0002-5211-411X

\section{Manoel Otávio da Costa Rocha}

Universidade Federal de Minas Gerais, Pós-Graduação em Ciências da Saúde: Infectologia e Medicina Tropical, Belo Horizonte, Minas Gerais, Brazil.

ORCID: 0000-0002-4721-1767

\section{INTRODUÇÃO}

A hanseníase é uma doença infecciosa causada pelo Mycobacterium leprae, também chamado de bacilo de Hansen, que atinge a pele e os nervos periféricos. A principal via de transmissão é pelas vias aéreas superiores. Esta doença é importante para a saúde pública, principalmente devido ao seu alto potencial de causar incapacidades físicas. A resposta imune do hospedeiro à infecção determinará o tipo de manifestação clínica, dependente do tipo de ativação linfocitária que leva a um espectro imunológico, clínico e histológico característico. ${ }^{1,2} \mathrm{M}$. leprae tem um tempo de multiplicação de 11 a 60 dias, considerado lento, o que justifica o tempo de incubação prolongado e a cronicidade da doença. Esse microrganismo é circundado por uma espessa parede lipídica de $20 \mathrm{~nm}$ de espessura, composta por polietilenoglicol-1 (PGL-1), que é específico desse patógeno. ${ }^{2}$

Em 2019, 202.185 casos de hanseníase foram notificados em todo o mundo. Índia e Brasil são os países com maior número de casos. ${ }^{3} \mathrm{O}$ diagnóstico tardio de hanseníase é uma preocupação no Brasil, uma vez que 10.813 novos casos de hanseníase foram identificados em 2019 já em grau 2 de incapa- cidade. O grau de deficiência é definido pela avaliação dos olhos, mãos e pés do paciente com intervalo de 0-2. No grau 0, os pacientes não apresentam alterações; no grau 1 , os pacientes diminuíram ou perderam a sensibilidade; no grau 2, os pacientes apresentam alterações nos olhos, como lagoftalmo e / ou ectrópio, triquíase. Nas mãos e nos pés, pode apresentar lesóes tróficas e / ou traumáticas; garras, reabsorção, pé caído e contratura do tornozelo. O esboço da Estratégia Global para a Hanseníase para o período de 20212030 visa eliminar a hanseníase até $2030 \mathrm{em}$ todo o mundo. Uma das estratégias para realizar o diagnóstico e o tratamento precoces é o rastreamento dos contatos de hanseníase. ${ }^{3}$

Os contatos podem ser definidos como pessoas que interagem com um paciente diagnosticado no ambiente doméstico, na vizinhança, na família ou no círculo social. 45,6 Estudos anteriores identificaram fatores de risco para adoecimento por contatos com hanseníase. Fatores de risco sociais, nutricionais, imunológicos, genéticos, bem como fatores relacionados ao caso índice, também foram descritos. ${ }^{7,8,9,10,11,12,13,14,15,16,17,18,19,20,21,22,23}$ Os seguintes fatores familiares e sociais foram descritos: consanguinidade com o caso índice, ${ }^{7}$ proximidade com o caso índice, ${ }^{7}$ vulnerabilidade social, ${ }^{7,89}$ deficiência nutricional, ${ }^{10,11}$ sexo e idade. ${ }^{12}$ Dentre os fatores imunológicos, ML Flow positivo, 13,14,15,16,17 ausência de cicatriz de BCG, ${ }^{18,19,20}$ e teste de Mitsuda negativo em comunicantes. ${ }^{12,17}$ Dentre os fatores genéticos, alguns autores descreveram: suscetibilidade hereditária ${ }^{21} \mathrm{e}$ estreita relação genética. ${ }^{22}$ Os fatores relacionados ao caso índice foram: ser multibacilar, ter índice baciloscópico alto e classificação de grau 2 de incapacidade. ${ }^{7,12,23} \mathrm{O}$ exame de contato é indicado para o manejo clínico e epidemiológico da doença.

A avaliação das evidências científicas ajuda a aprimorar o conhecimento sobre o risco de doenças no grupo de contato.

Um levantamento preliminar do PROSPERO, MEDLINE, o Cochrane Database of Systematic Reviews foi conduzido e não encontrou nenhuma revisão sistemática atual ou em andamento sobre o assunto. A última revisão com esta abordagem foi publicada em 2004. ${ }^{21}$ Este protocolo de revisão sistemática terá como objetivo avaliar os fatores de risco associados ao desenvolvimento da doença em contatos com hanseníase e a questão norteadora será: Quais são os fatores de risco associados ao desenvolvimento da doença em contatos de hanseníase? 


\section{MÉTODO}

A revisão sistemática será conduzida de acordo com a metodologia PRISMA-P para revisões sistemáticas de evidências de eficácia. ${ }^{25,26} \mathrm{O}$ protocolo de revisão sistemática foi registrado no PROSPERO, sob o código CRD42020160680. Esta revisão levará em consideração estudos de elegibilidade que investiguem contatos com hanseníase, incluindo contatos domiciliares e contatos sociais; de ambos os sexos, de qualquer idade e considerará estudos que comparem fatores de risco para hanseníase em contatos e fatores de proteção para contatos saudáveis.

Os estudos serão selecionados sem restrição de idioma no período de 2004 a 2020. Serão selecionados estudos experimentais, como ensaios clínicos randomizados e não randomizados controlados, estudos de coorte prospectivos e retrospectivos, estudos de caso-controle e estudos analíticos transversais, sem restrição de idioma. Artigos duplicados, revisão narrativa ou sistemática e meta-análise, estudos da população em geral, estudos de pacientes com hanseníase, relatos de casos de outras doenças infecciosas (agudas ou crônicas), séries de casos, entrevistas, cartas editoriais e estudos experimentais envolvendo animais serão excluídos. Os estudos publicados e não publicados serão avaliados para elegibilidade. ${ }^{25}$

A estratégia de busca foi focada em estudos publicados. Uma busca inicial na base de dados MEDLINE foi realizada para identificar artigos sobre o tema. As palavras do texto nos títulos e resumos de artigos relevantes e os termos de índice usados para descrever os artigos foram usados para desenvolver uma estratégia de busca completa para banco de dados e plataforma A estratégia de busca, incluindo todas as palavras-chave e termos de índice identificados, será adaptada para cada fonte de informação incluída (Apêndice I). As listas de referência de todos os estudos selecionados para avaliação crítica serão selecionadas para estudos adicionais. Estudos não publicados e literatura cinzenta também serão pesquisados em busca de estudos relevantes.

Consideraremos as seguintes bases de dados como fontes de informação: Biblioteca Virtual em Saúde (BVS), PubMed, Bibliote- ca Cochrane, EMBASE (OVID), Fontes de estudos não publicados e literatura cinzenta a serem pesquisadas incluem MedNar, OpenGrey, ProQuest Dissertations and Theses e busca manual. Após a pesquisa, todas as citações identificadas serão agrupadas e carregadas na Rayyan Systems Inc, na qual as duplicatas serão identificadas e removidas. Após um teste piloto, os títulos e resumos serão selecionados por dois revisores independentes para avaliação em relação aos critérios de inclusão para a revisão (ESA; ALGO). O texto completo das citações selecionadas será avaliado em detalhes em relação aos critérios de inclusão por dois revisores independentes (ESA; ALGO). Os motivos para a exclusão de estudos de texto completo que não atendam aos critérios de inclusão serão registrados e relatados na revisão sistemática. Quaisquer divergências entre os revisores em cada estágio do processo de seleção do estudo serão resolvidas por meio de discussão ou com um terceiro ou quarto investigador, se necessário (NSG). Os resultados da pesquisa e do processo de seleção e inclusão do estudo serão relatados na íntegra na revisão sistemática final e apresentados em um fluxograma acordo com o PRISMA-P. ${ }^{26}$

Para avaliação da qualidade metodológi$\mathrm{ca}$, os dois revisores independentes usarão os instrumentos padronizados para desenvolver uma avaliação crítica de estudos de caso-controle, transversais e de coorte, estudos prospectivos e retrospectivos para avaliar os fatores de risco relacionados ao contato da hanseníase no desenvolvimento da doença. ${ }^{22}$ Autores de artigos serão contatado para solicitar dados em falta ou adicionais para esclarecimentos em qualquer fase, se necessário. Quaisquer divergências entre os revisores serão discutidas e resolvidas. Os resultados da avaliação da qualidade metodológica dos estudos serão relatados em formato narrativo e adicionados a uma tabela. Todos os estudos, independentemente da qualidade metodológica dos resultados, serão submetidos à extração e síntese dos dados, quando possível. Os resultados da avaliação crítica por meio das ferramentas NEWCASTLE OTTAWA e ROBINS-I serão descritos na revisão de forma descritiva. Abordaremos como esses resultados podem influenciar a interpretação das evidências do estudo.
Serão considerados estudos que avaliam os seguintes desfechos: identificação de fatores associados ao desenvolvimento da doença em contatos com hanseníase. Fatores associados ao caso índice, como fatores sociais, nutricionais, genéticos e imunológicos, serão de interesse na revisão. Serão extraídas medidas de tempo e efeito, considerando os resultados obtidos nas análises de risco relativo, odds ratio, risco atribuível e demais medidas aplicáveis aos desfechos do estudo.

Os dados serão extraídos de estudos selecionados de forma independente por dois revisores (ESA; ALGO) usando o Rayyan Systems Inc. Os dados extraídos incluirão detalhes sobre a população, métodos de estudo, exposições e resultados, como a seguir:

- Detalhes do estudo: autor, ano de publicação, país.

- Método / características de estudo: o local de estudo e cenário de pesquisa; desenho do estudo e acompanhamento, população (número de participantes, sexo e idade); fatores de risco (todas as variáveis investigadas no estudo); fatores de proteção incluindo todas as variáveis investigadas no estudo; grupos comparativos;

- Resultados: fatores de proteção (todas as variáveis investigadas / exploradas no estudo); resultados principais (fatores de risco com significância estatística); resultados principais (fatores de proteção com significância estatística); casos detectados entre os contatos/incidência ou prevalência.

- Métodos de análise de dados: estimativas estatísticas expressas por razão de risco, razão de risco relativo, razão de chances, valores de $\mathrm{p}$ e intervalos de confiança (IC).

Quaisquer divergências que surjam entre os revisores serão resolvidas por meio de discussão ou com um terceiro revisor (NSG). Analisaremos os dados disponíveis e o impacto da falta de dados será considerado uma limitação do estudo, quando aplicável.

\section{RESULTADO}

Os dados serão extraídos dos estudos selecionados serão sintetizados em tabelas, 
e os resultados compilados serão considerados para o desenvolvimento de uma meta-análise. A síntese e a análise dos dados serão elaboradas para abordar a questão da pesquisa sobre os fatores de risco associados ao desenvolvimento da doença em contatos com hanseníase. Para cada estudo primário, OR e seu erro padrão serão extraídos. Para estudos com variáveis dicotômicas, serão obtidos a partir dos resumos dos modelos de regressão logística, testes qui-quadrado, ou teste exato de Fisher, ou quando não disponíveis diretamente, serão calculados a partir de dados brutos. Para estudos com variáveis contínuas, as estimativas de OR serão obtidas por meio de transformações dos resultados da regressão linear de acordo com a relação conhecida entre as distribuições normal e logística. Os resultados dos estudos serão agrupados e uma estimativa geral de OR será obtida a partir de modelos de efeitos aleatórios para explicar a heterogeneidade do estudo. Todas as estimativas do modelo serão mostradas com ICs de $95 \%$, e a estatística Q, a estatística I2 e a estatística tau-quadrada serão usadas para avaliar a heterogeneidade entre os estudos. Parcelas florestais para todas as meta-análises serão fornecidas.

$\mathrm{Na}$ presença de diferenças de subgrupo, métodos de meta-regressão serão usados para investigar os efeitos de covariáveis categóricas ou contínuas. $\mathrm{O}$ viés de publicação será investigado usando o gráfico de funil e o teste de regressão de Egger. Um valor de $\mathrm{p}$ $<0,05$ será considerado estatisticamente significativo. Todas as análises serão conduzidas usando Stata ou R. Se a meta-análise não for possível, os resultados serão apresentados em uma forma narrativa, incluindo tabelas e figuras para auxiliar na apresentação dos da- dos quando apropriado. Temas de interesse, como a identificação de fatores associados ao desenvolvimento da doença em contatos de hanseníase, serão identificados e discutidos. Faremos uma avaliação abrangente da qualidade metodológica dos estudos. A conclusão da revisão está prevista para julho de 2021. As atualizações de buscas serão realizadas, caso necessário.

\section{DISCUSSÃO}

Este estudo permitirá avaliar os fatores de risco relacionados ao desenvolvimento da doença em contatos de pacientes com hanseníase. Serão explorados os aspectos sociais, familiares, nutricionais, imunológicos, genéticos, bem como fatores relacionados ao caso índice, que possam estar associados como fatores de risco para adoecimento na população de contatos, e identificaremos fatores de proteção para o desenvolvimento da doença.

O controle dos contatos é um dos pilares estratégicos para que ocorra uma vigilância efetiva, ${ }^{27}$ mas é preciso realizar o controle de forma sistemática e direcionada para maior efetividade das ações de controle. Outros autores já reforçaram que é importante estruturar novos estudos como caminho para subsidiar o desenvolvimento de outras estratégias para o controle da hanseníase. ${ }^{28}$

O acompanhamento da população de alto risco é necessário para conseguir um efetivo controle da hanseníase. ${ }^{29}$ A sistematização do atendimento e a proposição de tecnologias leves para a atenção aos contatos de hanseníase torna-se essencial para o êxito no controle da doença e no diagnóstico precoce, auxiliando em um sistema de vigilância efetivo e gerenciamento de contatos. Espera-se oferecer uma abordagem que contribua para melhorar o conhecimento científico sobre o tema, fornecendo subsídios para estruturação de meios para melhor manejo do grupo de contatos de hanseníase.

A aplicação da saúde baseada em evidências permite explorar e delimitar o risco nesta população, pois a avaliação qualificada do corpo de evidências disponível pode-se identificar possíveis estratégias aplicáveis ao contexto da vigilância de contatos. A saúde baseada em evidências é definida como um uso consciente, explícito e criteriosos da melhor evidência na literatura. ${ }^{30}$

O potencial deste estudo consiste no preenchimento de uma lacuna do conhecimento científico referente aos determinantes de adoecimento no grupo de contatos e impacto da doença nesta população. É necessário identificar os fatores de risco para o desenvolvimento da doença para orientar o estabelecimento de estratégias de vigilância adequadas aos contatos de hanseníase. Os resultados da presente revisão sistemática poderão contribuir para o entendimento da epidemiologia da hanseníase e a proposição de intervenções em saúde pública. Espera-se que os resultados deste estudo estejam disponíveis em julho de 2021.

\section{CONCLUSÃO}

Espera-se que este trabalho contribua para a identificação e análise dos fatores de risco relacionados ao adoecimento em contatos de hanseníase. Monitorar os contatos é uma das estratégias mais eficazes para o controle da hanseníase. A identificação dos fatores de risco para adoecimento nos contatos pode contribuir para o desenvolvimento de ações em saúde pública e melhoria do processo assistencial. .

\section{REFERÊNCIAS}

1.Walker SL, Lockwood DNJ. 2007. Leprosy.Clin Dermatol25:16572. http://dx.doi.org/10.016/j.clindermatol. 2006.05.0122.Lyon S Grossi MA. Hanseníase. Rio de Janeiro: Medbook, 2013. 520p

3. World Health Organization. Weekly Epidemiological Record, 2019, vol. 94, 35/36 (pp. 389-412)

4. Moet FJ, Meima A, Oskam L, Richardus JH. Risk factors for the development of clinical leprosy among contacts, and their relevance for targeted interventions. Lep Rev. 2004b; 75:310-6.

5. Calado KLS, Vieira AG, Durães S, Sékula SB, Oliveira MLWDR. Positividade sorológica antiPGL-I em contatos domiciliares e peridomiciliares de hanseníase em área urbana. An. Bras. Dermatol. [Internet]. 2005 Dec [cited 2020 Oct 16]; 80( Suppl 3): S301-S306. [Cited 2019 10 05] Available from: http://www.scielo.br/scielo.php?script=sci_ 


\section{REFERÊNCIAS}

arttext\&pid=S0365-05962005001000007\&lng=en.

6. Brasil. Ministério da Saúde. Secretaria de Vigilância em Saúde. Departamento de Vigilância das Doenças Transmissiveis. Diretrizes para vigilância, atenção e eliminação da hanseníase como problema de saúde pública. Brasília: Ministério da Saúde, 2016.

7. Sales AM, Ponce de Leon A, Düppre NC, Hacker MA, Nery JA, Sarno EN, Penna ML. Leprosy among patient contacts: a multilevel study of risk factors. PLoS Negl Trop Dis. 2011;5(3):e1013.Erratum in: PLoS Negl Trop Dis. 2011;5(8).

8. Freitas LR, Duarte EC, Garcia LP. Leprosy in Brazil and its association with characteristics of municipalities: ecological study, 20092011. Trop Med Int Health. 2014 Oct; 19(10):1216-25.

9. White C, Franco-Paredes C. Leprosy in the 21st century. Clin Microbiol Rev. 2015;28:80-94.

10. Passos Vázquez CM, Mendes Netto RS, Ferreira Barbosa KB, Rodrigues de Moura T, de Almeida RP, Duthie MS, Ribeiro de Jesus A. Micronutrients influencing the immune response in leprosy. Nutr Hosp. 2014;29(1):26-36

11. SILVA, C.P.G; MIYAZAKI, M.C.O.S. Hanseníase e a nutrição: uma revisão da literatura. Hansen. int; 37(2): 69-74, 2012. [Cited 2018 0812]. Available from: http://periodicos.ses.sp.bvs.br/scielo. php? script=sci_ abstract\&pid=S1982-51612012000200009\&I$\mathrm{ng}=\mathrm{en \& nrm}=$ isso.

12. Sarno EN, Duppre NC, Sales AM, Hacker MA, Nery JA, Matos HJ. Leprosy exposure, infection and disease: a 25-year surveillance study of leprosy patient contacts. Mem. Inst. Oswaldo Cruz [Internet]. 2012.107(8):1054-1059. [cited 2019 Oct 16]. Available from: http://www.scielo.br/scielo.php?script=sci_arttext\&pid=S0074-02762012000800015\&lng=en.

13. Andrade AR, Grossi MA, Bührer-Sékula S, Antunes CM. Seroprevalence of ML Flow test in leprosy contacts from State of Minas Gerais, Brazil. Rev Soc Bras Med Trop. 2008;41:56-9//

14. Araújo S, Rezende MMF, Sousa DCR, Rosa MR, Santos DC, Goulart LR, et al. Risk-benefit assessment of Bacillus Calmette-Guérin vaccination, anti-phenolic glycolipid I serology, and Mitsuda test response: 10-year follow-up of household contacts of leprosy patients. Rev. Soc. Bras. Med. Trop. 2015;48(6):739-745.

15. Cardona-Castro N, Beltrán-Alzate JC, Manrique-Hernández R. Survey to identify Mycobacterium leprae-infected household contacts of patients from prevalent regions of leprosy in Colombia. Mem Inst Oswaldo Cruz. 2008;103(4):332-6.

16. Düppre NC, Camacho LA, Sales AM, Illarramendi X, Nery JA, Sampaio EP, Sarno EN, Bührer-Sékula S. Impact of PGL-I seropositivity on the protective effect of BCG vaccination among leprosy contacts: a cohort study. PLoS Negl Trop Dis. 2012;6(6):e171.

17. Goulart IM, Bernardes Souza DO, Marques CR, Pimenta VL, Gonçalves MA, Goulart LR. Risk and protective factors for leprosy development determined by epidemiological surveillance of household contacts. Clin Vaccine Immunol. 2008 ;15(1):101-5.

18. Britton WJ, Lockwood DN. Leprosy. Lancet. 2004;363(9416):1209-19
19. Merle CS, Cunha SS, Rodrigues LC. BCG vaccination and leprosy protection: review of current evidence and status of BCG in leprosy control. Expert Rev Vaccines. $2010 ; 9(2): 209-22$.

20. Richardus JH, Oskam L. Protecting people against leprosy: chemoprophylaxis and immunoprophylaxis. Clin Dermatol. 2015;33(1):19-25

21. Moet FJ, Meima A, Oskam L, Richardus JH. Risk factors for the development of clinical leprosy among contacts, and their relevance for targeted interventions. Lepr Rev. 2004 Dec;75(4):310-26. PMID: 15682969.

22 Moet FJ, Pahan D, Schuring RP, Oskam L, Richardus JH. Physical distance, genetic relationship, age, and leprosy classification are independent risk factors for leprosy in contacts of patients with leprosy. J Infect Dis. 2006 Feb 1;193(3):346-53

23. Carvalho AP, da Conceição Oliveira Coelho Fabri A, Corrêa Oliveira R, Lana FC. Factors associated with anti-phenolic glycolipid-I seropositivity among the household contacts of leprosy cases. BMC Infect Dis. 2015;15:219.

24. Alecrim ES, Chaves AT, Pôrto LAB, Grossi MAF, Lyon S, Rocha MODC. Reading of the Mitsuda test: comparison between diameter and total area by means of a computerized method. Rev Inst Med Trop Sao Paulo. 2019 Feb 14;61:e5.

25. Shamseer L, Moher D, Clarke M, Ghersi D, Liberati A, Petticrew M, Shekelle P, Stewart L, PRISMA-P Group. Preferred reporting items for systematic review and meta-analysis protocols (PRISMA-P) 2015: elaboration and explanation. BMJ. 2015 Jan 2;349(jan02 1):g7647.

26. Moher D, Shamseer L, Clarke M, Ghersi D, Liberati A, Petticrew $M$, Shekelle P, Stewart LA. Preferred Reporting Items for Systematic Review and Meta-Analysis Protocols (PRISMA-P) 2015 statement. Syst Rev. 2015;4(1):1.

27. Gomes da Silva CF, Toneli Nardi S, Wilian Lozano A, Martins Pinto Neto J, Rodrigo Ramos R, Del'Arco Paschoal V. Fatores de risco para hanseníase identificados a partir de casos notificados num período de 15 anos. SaudColetiv (Barueri) [Internet]. $24^{\circ}$ de novembro de 2020 [citado $15^{\circ}$ de maio de 2021];10(58):3789-802.

28 Silva MA, dos Santos Lago K, Martins da Costa C, de Caux Bueno I, Nogueira Rodrigues R. Tendência da situação epidemiológica e operacional da hanseníase no estado de Minas Gerais (2008 - 2018). SaudColetiv (Barueri) [Internet]. $29^{\circ}$ de setembro de 2020 [citado $15^{\circ}$ de maio de 2021];10(56):3122-31.

29. Cardona-Castro Nora M, Restrepo-Jaramillo Sandra, Gil de la Ossa Myriam, Brennan Patrick J. Infection by Mycobacterium leprae of household contacts of lepromatous leprosy patients from a post-elimination leprosy region of Colombia. Mem. Inst. Oswaldo Cruz [Internet]. 2005 Nov [cited 2021 May 15] ; 100( 7 ): 703-707. Available from: http://www.scielo.br/scielo.php?script=sci_arttext\&pid=S0074-02762005000700003\&Ing=en.

30. Barini, Orestes. Medicina baseada em evidências. Rev. Assoc. Med. Bras. [Internet]. 2001 Dec [cited 2021 May 15] ; 47( 4): 294294. Available from: http://www.scielo.br /scielo . php?script=sci _artt ext\&pid=S0104-42302001000400029\&Ing=en. 
Apêndice I: Estratégias de pesquisa para BVS (BVS), MEDLINE (PUBMED), CINAHL (EBSCO), Web of Science (ISI), Cochrane Library, and Embase (OVID) databases

\begin{tabular}{|c|c|c|}
\hline BASES & ESTRATÉGIAS & REGISTROS RECUPERADOS \\
\hline BVS & $\begin{array}{l}\text { tw:((tw: leprosy OR lepra OR hanseníase OR lepra OR "Doença de Hansen" } \\
\text { OR "Hansen Disease" OR "Disease, Hansen" OR "Disease, Hansen's" } \\
\text { OR "Hansens Disease" OR leprosies OR "Hansen's Disease") AND (tw: } \\
\text { "Contact Tracing" OR "Trazado de Contacto" OR "Busca de Comunicante" } \\
\text { OR "Busca de Comunicante de Doenças Transmissiveis" OR "Busca de } \\
\text { Comunicante de Doenças Infecciosas" OR "Notificação do Parceiro" OR } \\
\text { "Notifications, Partner" OR "Partner Notifications" OR "Tracing, Contact" } \\
\text { OR "Communicable Disease Contact Tracing" OR "Infectious Disease } \\
\text { Contact Tracing" OR "Partner Notification" OR "Notification, Partner" } \\
\text { OR "Epidemiological Monitoring" OR "Monitoreo Epidemiológico" OR } \\
\text { "Monitoramento Epidemiológico" OR "Epidemiologic Monitoring" OR } \\
\text { "Epidemiologic Surveillance" OR "Monitoring, Epidemiologic" OR "Epide- } \\
\text { miologic Surveillances" OR "Monitoring, Epidemiological" OR "Surveillan- } \\
\text { ce, Epidemiologic" OR "Surveillances, Epidemiologic" OR "Epidemiological } \\
\text { Surveillance" OR monitoring OR monitoreo OR monitoramento OR con- } \\
\text { tact* OR contato*) AND (tw: risk OR riesgo OR risco OR "Relative Risks" } \\
\text { OR "Risk, Relative" OR risks OR "Risks, Relative" OR "Relative Risk" OR } \\
\text { "Risk Factors" OR "Factores de Riesgo" OR "Fatores de Risco" OR "Popula- } \\
\text { tion at Risk" OR "Populations at Risk" OR "Factor, Risk" OR "Factors, Risk" } \\
\text { OR "Risk Factor" OR "Risk, Population at" OR "Risk, Populations at" OR } \\
\text { "Protective Factors" OR "Factores Protectores" OR "Fatores de Proteção" } \\
\text { OR "Fatores Protetores" OR "Factor, Protective" OR "Factors, Protective" } \\
\text { OR "Protective Factor" OR "Risk Assessment" OR "Medición de Riesgo" } \\
\text { OR "Medição de Risco")) AND (instance:"regional") AND ( db:"LILACS" OR } \\
\text { "WHOLIS" OR "HANSENIASE" OR "IBECS" OR "SES-SP" OR "BDENF" OR } \\
\text { "CUMED" OR "BINACIS")) }\end{array}$ & 199 \\
\hline Medline via PUBMED & $\begin{array}{c}\text { (((("Leprosy"[Mesh]) OR "Leprosy"[Title/Abstract])) AND (((("Contact Tra- } \\
\text { cing"[Mesh]) OR "Epidemiological Monitoring"[Mesh])) OR ("Contact Tra- } \\
\text { cing"[Title/Abstract] OR "Epidemiological Monitoring"[Title/Abstract] OR } \\
\text { Monitoring[Title/Abstract] OR Contact*[Title/Abstract]))) AND (((((("Risk"[- } \\
\text { Mesh]) OR "Risk Factors"[Mesh]) OR "Protective Factors"[Mesh]) OR "Risk } \\
\text { Assessment"[Mesh])) OR ("Risk"[Title/Abstract] OR "Risk Factors"[Title/ } \\
\text { Abstract] OR "Protective Factors"[Title/Abstract] OR "Risk Assessment"[- } \\
\text { Title/Abstract])) }\end{array}$ & 257 \\
\hline Cochrane Central & $\begin{array}{c}\text { (Leprosy OR "Hansen Disease" OR "Disease, Hansen" OR "Disease, } \\
\text { Hansen's" OR "Hansens Disease" OR Leprosies OR "Hansen's Disease") } \\
\text { AND ("Contact Tracing" OR "Notifications, Partner" OR "Epidemiological } \\
\text { Monitoring" Monitoring OR Contact*) AND (Risk OR "Relative Risks" OR } \\
\text { "Factor, Risk" OR "Protective Factors" OR "FactoresProtectores" OR "Risk } \\
\text { Assessment") }\end{array}$ & 19 \\
\hline Embase & $\begin{array}{c}\text { ('leprosy'/exp OR leprosy) AND ('contact examination'/exp OR 'contact } \\
\text { examination' OR 'contact tracing'/exp OR 'contact tracing' OR 'epidemio- } \\
\text { logical monitoring'/exp OR 'epidemiological monitoring' OR 'monitoring'/ } \\
\text { exp OR monitoring OR 'contact'/exp OR contact) AND ('risk'/exp OR risk } \\
\text { OR 'risk factor'/exp OR 'risk factor' OR 'protection'/exp OR protection OR } \\
\text { 'protective factors'/exp OR 'protective factors' OR 'risk assessment'/exp } \\
\text { OR 'risk assessment') }\end{array}$ & 69 \\
\hline
\end{tabular}

